\title{
Tacrolimus Monotherapy: A Promising Option for Ocular Myasthenia Gravis
}

\author{
Yohsuke Yagi Nobuo Sanjo Takanori Yokota Hidehiro Mizusawa \\ Department of Neurology and Neurological Science, Graduate School of Medical and Dental Sciences, \\ Tokyo Medical and Dental University, Tokyo, Japan
}

Dear Sir,

Myasthenia gravis (MG) is an autoimmune disease that affects the neuromuscular junction. Anticholinesterase agents are the basic symptomatic treatment for $\mathrm{MG}$, which partially compensates for the reduced safety margin at the neuromuscular junction; these agents may be sufficient in rare cases of mild MG with purely ocular involvement. Corticosteroid immunosuppressant drugs are the mainstay of diseasemodifying therapy for MG; however, the adverse effects of corticosteroids include osteoporosis, hypertension, exacerbation or precipitation of diabetes mellitus, gastrointestinal ulcers, cataracts, and opportunistic infection [1]. Therefore, an alternative to corticosteroid therapy is needed, especially in elderly patients. The calcineurin inhibitor tacrolimus is the immunosuppressant agent used for organ transplantation and a variety of immune-mediated disorders including MG. Currently, tacrolimus is recommended only as a third-line therapy for long-term use in patients who are unresponsive to azathioprine, methotrexate, or mycophenolate mofetil [2]. To our knowledge, several preceding reports have documented the efficacy of tacrolimus for MG when combined with corticosteroids [3-5], but we are not aware of other studies that documented the efficacy of tacrolimus alone for MG.
We reviewed the records of patients with ptosis-onset ocular MG at the Tokyo Medical and Dental University Hospital from January 2005 until November 2010. Patients who took other immunosuppressive agents or underwent thymectomy were excluded. The clinical features of 4 MG patients who were treated with a cholinesterase inhibitor and tacrolimus as the only immunosuppressant agent are shown in table 1 . The dose of cholinesterase inhibitor was fixed during the observation. The mean (SD) age of the patients was 74.0 (9.3) years and the male-to-female ratio was 2:2. All of the patients were Myasthenia Gravis Foundation of America clinical classification class I and had responded well to tacrolimus. We observed these patients for 24 months after starting tacrolimus. The mean anti-acetylcholine receptor antibody titers before and after the treatment with tacrolimus were 26.35 (24.7) and 13.73 (15.6) nmol/l, respectively. The antiacetylcholine receptor antibody titer was reduced in all patients after the treatment, and the posttreatment-to-pretreatment ratio was 0.50 (0.20). Remarkable adverse effects were not observed during tacrolimus administration.

All 4 patients responded well to tacrolimus without prednisolone or other immunosuppressive agents and have remained stable for longer than 2 years (ocular-quantitative MG (QMG) score in table 1). The ocular-QMG score is used to evaluate ocular symptoms in myasthenia and includes 3 of the 13 items from the QMG scale [6]. There was no constant tendency regarding which ocular symptom reacted better to tacrolimus; ptosis or diplopia. Several researchers reported that the anti-acetylcholine receptor antibody titer or its ratio is a marker for the efficacy of calcineurin inhibitors, including tacrolimus $[7,8]$. In our cases, the mean anti-acetylcholine receptor antibody ratio was reduced after the tacrolimus treatment and was similar to the values reported previously [7]. This suggests that the anti-acetylcholine receptor antibody titer or its ratio is a hallmark of the response to tacrolimus.

The mean time period from disease onset to the time when tacrolimus was started was 17.25 (14.0) months, suggesting that MG patients who have a long disease course can respond well to tacrolimus. Because of the lack of high-quality evidence for the treatment of ocular MG, there is no evidence-based consensus regarding the effects of cholinesterase inhibitors, corti-

Y.Y. and N.S. contributed equally to this work.

\section{KARGER}

E-Mail karger@karger.com

www.karger.com/ene (c) 2013 S. Karger AG, Base

0014-3022/13/0696-0344\$38.00/0
Takanori Yokota

Department of Neurology and Neurological Science, Graduate School of Medical and Dental Sciences, Tokyo Medical and Dental University, 1-5-45 Yushima, Bunkyo-ku Tokyo 1138519 (Japan)

E-Mail yokonuro@tmd.ac.jp 
Table 1. Clinical features of the 4 cases

\begin{tabular}{|c|c|c|c|c|}
\hline & Case 1 & Case 2 & Case 3 & Case 4 \\
\hline Age at onset of MG, years & 77 & 83 & 61 & 75 \\
\hline Gender & F & $\mathrm{F}$ & M & M \\
\hline MGFA clinical classification & class I & class I & class I & class I \\
\hline Ptosis & + & + & + & + \\
\hline Diplopia & + & - & + & + \\
\hline \multicolumn{5}{|l|}{ Ocular-QMG score } \\
\hline Before starting tacrolimus & 6 & 3 & 6 & 6 \\
\hline At 1 month & 0 & 0 & 3 & 0 \\
\hline At 2 months & 0 & 0 & 0 & 0 \\
\hline At 3 months & 0 & 0 & 0 & 0 \\
\hline At 6 months & 0 & 0 & 0 & 0 \\
\hline At 12 months & 0 & 0 & 0 & 0 \\
\hline At 24 months & 0 & 0 & 0 & 0 \\
\hline \multicolumn{5}{|l|}{ Time period from disease onset to the time } \\
\hline when tacrolimus was started, months & 22 & 6 & 35 & 6 \\
\hline Maintenance dose of tacrolimus, mg & 2 & 1.5 & 3 & 3 \\
\hline Trough concentration of tacrolimus, $\mathrm{ng} / \mathrm{ml}$ & 4.0 & 8.2 & 5.5 & 5.3 \\
\hline Anti-AChR Ab before starting tacrolimus, $\mathrm{mmol} / \mathrm{l}$ & 63 & 9.4 & 17 & 16 \\
\hline Anti-AChR Ab after starting tacrolimus, nmol/l & 37 & 7.2 & 6.6 & 4.1 \\
\hline Anti-AChR Ab ratio & 0.59 & 0.77 & 0.39 & 0.26 \\
\hline
\end{tabular}

MGFA $=$ Myasthenia Gravis Foundation of America; $\mathrm{AChR}=$ acetylcholine receptor.

costeroids, or other immunosuppressive agents with respect to improvement of ocular symptoms [9]. In this study, 4 patients did not receive steroids because of their age or other complications such as gastrointestinal tract bleeding, not because they had milder symptoms. From January 2005 until November 2010, we treated 23 ocular MG patients including the 4 patients presented here. These 4 patients treated with tacrolimus alone had a higher ocular-QMG score (median value 6) compared with the other 19 patients (median value $3 ; \mathrm{p}=0.067$, Mann-Whitney U).

Although this study was restricted to ocular MG patients, previous studies have demonstrated the efficacy of tacrolimus as part of a combination therapy for generalized MG. Therefore, the efficacy of tacrolimus monotherapy should be investigated in generalized MG. In conclusion, because tacrolimus monotherapy appears to be a safe and effective treatment option for ocular MG, it can be recommended as the initial treatment for this condition, especially in elderly patients. Large randomized controlled trials are needed to confirm this observation.

\section{Disclosure Statement}

This study had no sponsorship.

\section{References}

1 Gold R, Schneider-Gold C: Current and future standards in treatment of myasthenia gravis. Neurotherapeutics 2008;5:535-541.

12 Sathasivam S: Steroids and immunosuppressant drugs in myasthenia gravis. Nat Clin Pract Neuro 2008;4:317-327.

-3 Ponseti JM, Azem J, Fort JM, López-Cano M, Vilallonga $\mathrm{R}$, Buera $\mathrm{M}$, et al: Long-term results of tacrolimus in cyclosporine- and prednisone-dependent myasthenia gravis. Neurology 2005;64:1641-1643.

4 Schneider-Gold C, Hartung HP, Gold R: Mycophenolate mofetil and tacrolimus: new therapeutic options in neuroimmunological diseases. Muscle Nerve 2006;34:284-291.
5 Konishi T, Yoshiyama Y, Takamori M, Yagi K, Mukai E, Saida T; Japanese FK506 MG Study Group: Clinical study of FK506 in patients with myasthenia gravis. Muscle Nerve 2003;28:570-574.

6 Bhanushali MJ, Wuu J, Benatar M: Treatment of ocular symptoms in myasthenia gravis. Neurology 2008;71:1335-1341.

-7 Nagane Y, Suzuki S, Suzuki N, Utsugisawa K: Factors associated with response to calcineurin inhibitors in myasthenia gravis. Muscle Nerve 2010;41:212-218.
-8 Ponseti JM, Gamez J, Azem J, López-Cano M, Vilallonga R, Armengol M: Tacrolimus for myasthenia gravis: a clinical study of $212 \mathrm{pa}-$ tients. Ann NY Acad Sci 2008;1132:254-263.

-9 Benatar M, Kaminski HJ; Quality Standards Subcommittee of the American Academy of Neurology: Evidence report: the medical treatment of ocular myasthenia (an evidencebased review): report of the Quality Standards Subcommittee of the American Academy of Neurology. Neurology 2007;68: 2144-2149. 